\title{
Survival after Complete Laryngotracheal Separation: A Case Report
}

\author{
Kimberly Serbousek, Eun Hae Estelle Chang and Christopher Bingcang \\ Department of Otolaryngology Head and Neck Surgery, University of Nebraska Medical Center
}

Submission: October 11, 2016; Published: October 20, 2016

*Corresponding author: Kimberly Serbousek, Eun Hae Estelle Chang and Christopher Bingcang, Department of Otolaryngology Head and Neck Surgery, University of Nebraska Medical Center, 981225 Nebraska Medical Center, Omaha, NE 68198-1225, USA.

\begin{abstract}
Objectives: 1) to describe a rare case of complete laryngotracheal separation (LTS) after attempted suicide by hanging. 2) To discuss the diagnosis and management of complete laryngotracheal separation.

Methods: A detailed chart review of the patient's medical and surgical management was performed.

Results: Successful treatment of complete laryngotracheal separation with primary anastamosis was accomplished. Complications including subglottic stenosis and bilateral vocal cord immobility were managed and stable long term outcomes achieved.

Conclusion: Trauma to the larynx occurs in varying degrees of severity, the highest of which results in complete laryngotracheal separation. Laryngotracheal separation often presents with non-specific symptoms in the setting of an acute trauma to the neck, which can lead to a delayed or missed diagnosis.
\end{abstract}

Keywords: Laryngotracheal separation; Laryngeal trauma; Hanging; Trauma

Abbreviations: LTS: Laryngotracheal separation; ET: Endotracheal Tube; CT: Computed Tomography

\section{Introduction}

Suicide attempts by hanging apply a lower magnitude force to the neck compared to judicial hangings [1]. Death is usually not immediate and occurs through asphyxiation, arterial occlusion, or venous occlusion [1]. If the patient survives following a suicide attempt via hanging, there is usually significant damage to the airway including the rare possibility of complete Laryngotracheal separation. Laryngotracheal separation (LTS) is not always obvious to diagnose clinically and health care providers must have a high degree of suspicion. We discuss a case presentation and management of complete LTS after attempted suicide by hanging.

\section{Case Presentation}

A 17-year-old male was injured in an unsuccessful suicide attempt by hanging from a bridge approximately 20 miles from the nearest hospital. The rope broke and he fell into the water below. He was found near the highway confused with a large neck laceration. Emergency medical services team arrived to the scene, he became obtunded and initial attempts to intubate the patient were unsuccessful. His airway was maintained via bag-mask-ventilation until the life flight team arrived. They were able to intubate the patient with a 7.0 endotracheal tube. He was transferred via air flight to a nearby level 1 trauma hospital. The initially documented injuries included bilateral pneumothoraces, right common carotid dissection, and the presence of subcutaneous emphysema. Chest tubes were placed and he was started on a heparin drip.

On the day of admission, he was alert and following commands, and he was considered to be ready for extubation. Shortly after extubation, he became stridulous and required emergent reintubation resulting in significant subcutaneous emphysema. A bronchoscopy was performed which revealed presence of edematous hypopharyngeal structures and suboptimal placement of the endotracheal (ET) tube. No false passages were found. The ET tube was repositioned and adequate oxygenation obtained. Over the next few days extubation was attempted three more times but failed with similar results. The patient was subsequently transferred to our facility for further care under our specialty trained laryngologist (senior author).

A direct laryngoscopy was performed showing normal anatomy of the supraglottis and glottis. The endotracheal tube 
was removed under direct visualization. Bronchoscopy was performed which revealed an approximately $3.5 \mathrm{~cm}$ separation between the cricoid ring and the distal trachea. Based on this finding, he was re-intubated. The patient was taken back to the operating room the following day for neck exploration, laryngotracheal reconstruction, and tracheostomy. During open exploration, the recurrent laryngeal nerves were not visualized secondary to diffuse inflammation. The thyroid cartilage was found to be intact.

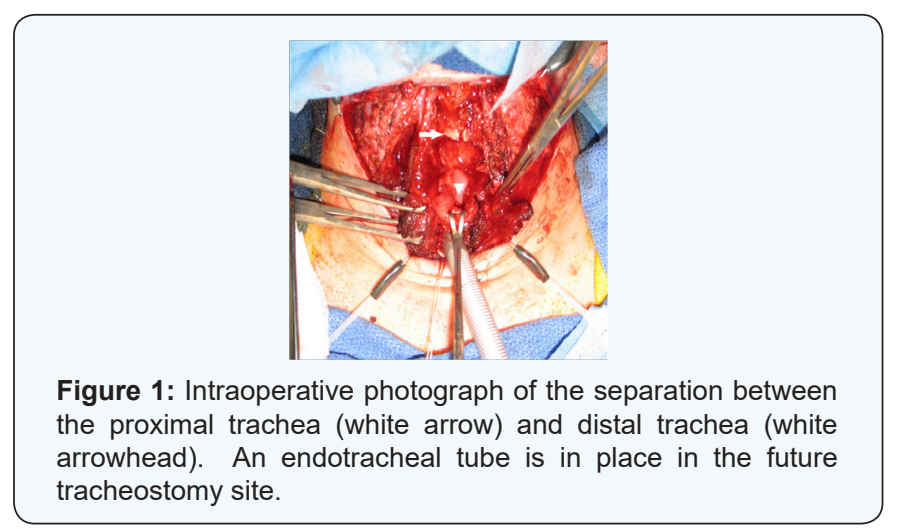

However, an anterior comminuted fracture in the cricoid cartilage was present as well as a $4 \mathrm{~cm}$ separation between the cricoid cartilage and distal trachea (Figure 1). After debriding inflamed mucosa from the subglottic larynx the trachea and cricoid were reapproximated and successful anastomosis was achieved by telescoping the proximal trachea into the subglottic larynx. A 6.0 cuffed Shiley tracheostomy tube was placed below the anastomosis site given the expected amount of swelling and inflammation in the subglottis.

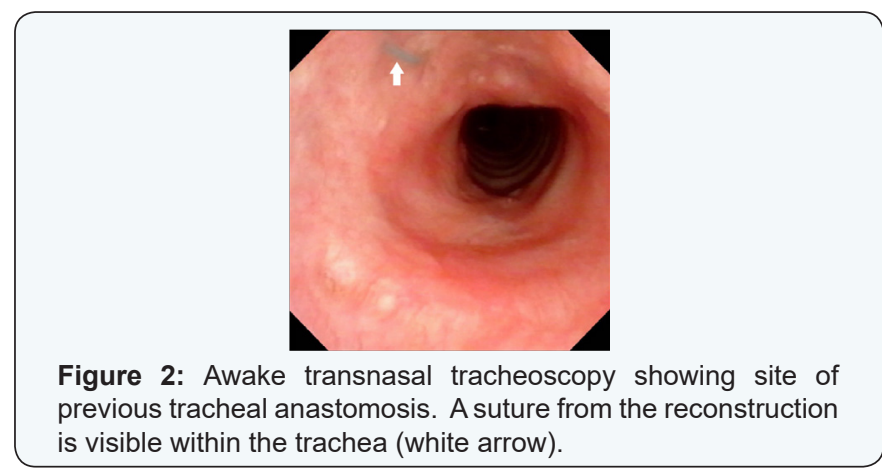

The patient returned to the operating room for repeat direct laryngoscopy on post reconstruction days 4, 14, and 27. He initially had moderate granulation tissues at the site of anastamosis, which resolved with repeat steroid injections. He was discharged home 46 days following tracheal reconstruction with a 6.0 Jackson tracheotomy tube in place. He later developed subglottic Stenosis at the anastamosis site requiring repeat balloon dilations and steroid injections. He had bilateral vocal cord immobility secondary to his initial injury and subsequently underwent vocal cord lateralization and a lateral cordotomy, which allowed him to be decanulated approximately 4 months after his reconstruction. He underwent scar revision on his anterior neck 9 months after LTS repair. He is currently 21 months status post reconstruction and his airway remains stable (Figure 2).

\section{Discussion}

Trauma to the larynx occurs in varying degrees of severity, the highest of which results in LTS [2] described as a grade 5 injuries by the Schaefer Classification System [3]. When a grade 5 injury occurs it carries a high rate of mortality [4]. LTS often presents with non-specific symptoms in the setting of an acute trauma to the neck [5], which can lead to a delayed or missed diagnosis. Dysphonia, Dyspnea, hemoptysis, and skin abrasions on the anterior neck are among the list of symptoms mentioned in the literature [6,7]. For instance, a case presentation by Choi et al. [8] reports hoarseness as the sole symptom of complete LTS after an attempted suicide by hanging. However, suspicion for laryngeal injury should be raised in the presence of subcutaneous emphysema.

The incidence of LTS is reported at less than $1 \%$ of laryngeal trauma [7], thus treatment algorithms or guidelines are not well established. The initial priority as in any trauma patient is to secure the airway. When there is a suspicion for LTS, the recommended first-line management is tracheotomy as intubation may create a false passage at the site of separation [9-11]. In this reported case, the patient was found to be in severe respiratory distress and was successfully intubated at the scene given the low degree of suspicion by initial primary responders. Once the airway is secured, computed tomography (CT) scan is a sensitive diagnostic test for laryngotracheal injury [12]. Retrospectively the CT images from the outside facility display a clear picture of the endotracheal balloon spanning the gap between the cricoid cartilage and distal tracheal (Figure 3).

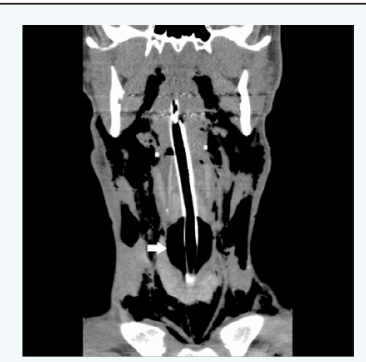

Figure 3: Coronal computed tomography showing the balloon of the endotracheal tube (white arrow) spanning the separation between the proximal and distal trachea. Subcutaneous air is present throughout the neck.

\section{Conclusion}

The current literature supports early surgical repair to decrease the incidence of subglottic Stenosis and to improve airway as well as voice outcomes $[13,14]$. Our report documents successful treatment with repeat balloon dilation along with steroid injection to treat the subglottic Stenosis complication. 
Additionally, temporary or permanent damage may occur to one or both of the recurrent laryngeal nerves due to LTS [9]. Future procedures including vocal cord lateralization with or without cordotomy may allow the patient to be decannulated if the nerve injury is deemed to be permanent. A high level of suspicion for LTS should be raised when direct trauma occurs to the larynx regardless of the presenting symptoms. If the patient does survive following LTS, numerous procedures are often necessary and a long term treatment plan must be developed.

\section{Consent}

Written informed consent was obtained from the patient and his parent for publication of this Case Report and any accompanying images. A copy of the written consent is available if required for review by the Editor-in-Chief of this journal.

\section{References}

1. Linnau K, Cohen W (2002) Radiologic evaluation of attempted suicide by hanging: cricotracheal separation and common carotid artery dissection. ARJ Am J Roentgenol 178(1): 214.

2. G. Richard Holt, Joseph A Brenna (2012) American Academy of Otolaryngology - Head and Neck Surgery Foundation Resident Manual of Trauma to the Face, Head, and Neck, (1 ${ }^{\text {st }}$ edn). Texas, USA.

3. Schaefer SD, Close LG (1989) Acute management of laryngeal trauma. Update. Ann Otol Rhinol Laryngol 98(2): 98-104.

4. Singh GB, Kummar R, Verma N, and Rai AK (2012) A rare case of survival after complete traumatic cricotracheal separation. Case Rep Otolaryngol 2012: 105763.
5. Aouad R, Moutran H, Rassi S (2007) Laryngotracheal disruption after blunt neck trauma. Am J Emerg Med 25(9): 1084e1-1084e2.

6. Borowski DW, Mehrotra P, Tennant D, El Badawey MR, Cameron DS (2004) Unusual presentation of blunt laryngeal injury with cricotracheal disruption by attempted hanging: a case report. Am J Otolaryngol 25(3): 195-198.

7. Singh B, Kumar S, Kumar V (2003) Blunt laryngotracheal injury following accidental strangulation. Injury 34(12): 937-939.

8. Choi JW, Koo BS, Rha KS, Yoon YH (2012) Complete Laryngotracheal Separation Following Attempted Hanging. Clinical and Experimental Otorhinolaryngology 5(3): 177-180.

9. McCrystal DJ, Bond C (2006) Cricotracheal separation: a review and a case with bilateral recovery of recurrent laryngeal nerve function. J Laryngol Otol 120(6): 497-501.

10. Costache VS, Renaud C, Brouchet L, Toma T, Le Balle F, et al. (2004) Complete tracheal rupture after a failed suicide attempt. Ann Thorac Surg 77(4): 1422-1423.

11. Baumgartner FJ, Ayres B, Theuer C (1997) Danger of false intubation after traumatic tracheal transection. Ann Thorac Surg 63(1): 227-228.

12. Francis S, Gaspard DJ, Rogers N, Stain SC (2002) Diagnosis and management of laryngotracheal trauma. J Natl Med Assoc 94(1): 2124.

13. Leopold DA (1983) Laryngeal trauma: a historical comparison of treatment methods. Arch Otolaryngol 109(2): 106-112.

14. Schaefer SD (1982) Primary management of laryngeal trauma. Ann Otol Rhinol Laryngol 91(4 Pt 1): 399-402. 